\title{
Design, capabilities and first results of the new laser blow-off system on Wendelstein 7-X
}

Th. Wegner, ${ }^{1}{ }^{1 \text { a) }}$ B. Geiger, ${ }^{1}$ F. Kunkel, ${ }^{1}$ R. Burhenn, ${ }^{1}$ T. Schröder, ${ }^{1}$ C. Biedermann, ${ }^{1}$ B. Buttenschön, ${ }^{1}$ G. Cseh, ${ }^{2}$ P. Drews,${ }^{3}$ O. Grulke, ${ }^{1}$ K. Hollfeld ${ }^{3}$ C. Killer, ${ }^{1}$ G. Kocsis, ${ }^{2}$ T. Krings,${ }^{3}$ A. Langenberg, ${ }^{1}$ O. Marchuk ${ }^{3}$ U. Neuner, ${ }^{1}$ D. Nicolai ${ }^{3}$ G. Offermanns, ${ }^{3}$ N. A. Pablant,${ }^{4}$ K. Rahbarnia, ${ }^{1}$ G. Satheeswaran, ${ }^{3}$ J. Schilling, ${ }^{1}$ B. Schweer, ${ }^{3}$ T. Szepesi, ${ }^{2}$ H. Thomsen, ${ }^{1}$ and W7-X Team ${ }^{1, b)}$

${ }^{1)}$ Max-Planck Institute for Plasma Physics, Greifswald, Germany

${ }^{2)}$ Wigner Research Center for Physics, Budapest, Hungary

${ }^{3)}$ Forschungszentrum Jülich, Jülich, Germany

${ }^{4)}$ Princeton Plasma Physics Laboratory, Princeton, NJ, USA

We present a detailed overview and first results of the new laser blow-off system on the stellarator Wendelstein 7-X. The system allows impurity transport studies by the repetitive and controlled injection of different tracer ions into the plasma edge. A Nd:YAG laser is used to ablate a thin metal film, coated on a glass plate, with a repetition rate of up to $20 \mathrm{~Hz}$. A remote-controlled adjustable optical system allows the variation of the laser spot diameter and enables the spot positioning to non-ablated areas on the target between laser pulses. During first experiments, clear spectral lines from higher ionization stages of the tracer ions have been observed in the X-ray to XUV spectral range. The temporal behavior of the measured emission allows the estimate of transport properties, e.g., impurity transport times in the order of $100 \mathrm{~ms}$. Although the strong injection of impurities is well detectable, the global plasma parameters are barely changed.

Keywords: stellarator, laser blow-off, laser ablation, impurity transport, impurity confinement, transport time

\section{INTRODUCTION}

The understanding of impurity transport in magnetic confined fusion devices is one of the high level objectives, especially for stellarators which are designed for steady state operation. Neoclassical transport calculations predict, that in the standard case of stellarators with negative radial electric field, the so-called ion-root regime, the collisional transport often may lead to impurity accumulation ${ }^{1}$ depending on the conditions, e.g., mean free path and temperature profile ${ }^{2}$. The existence and accumulation of highly charged impurities could lead to a decreased plasma performance or early pulse termination by increased radiative losses. It is therefore important to develop favorable operating scenarios and tools for a stationary plasma operation without impurity accumulation. Therefore, the transport quantities of intrinsic as well as artificially injected impurities are investigated. The injection of tracer impurity ions is advantageous because the impurity source is well known in terms of the injected amount and material as well as the spatial and temporal localization. Several methods are established for the injection of impurities, namely impurity pellet injection ${ }^{3,4}$, insertion of erosion probes $^{5}$, application of gas puffs and the laser blow-off technique ${ }^{6}$.

The laser blow-off (LBO) technique is a wide-spread method. It is not only used for impurity studies in fusion experiments ${ }^{6-14}$, but has also been applied for studying electron heat transport ${ }^{15-17}$, for measuring plasma temperatures and densities at the plasma edge ${ }^{18-23}$, investigating atomic processes $^{24-31}$ as well as laser induced metal deposition ${ }^{32}$ and patterning of thin film solar cells $\mathrm{s}^{33,34}$.

\footnotetext{
a)Electronic mail: thomas.wegner@ipp.mpg.de,physics@thwegner.com

b) R.C. Wolf et al. Nuclear Fusion 57, 102020 (2017)
}

In Wendelstein 7-X stellarator (W7-X) a new LBO system has been installed in 2017 for the OP1.2a experimental campaign to inject non-intrinsic and non-recycling impurity ions with different nuclear charge $Z$ for transport and confinement studies. The method is based on a high energy laser beam that passes through a transparent glass plate which is coated on the plasma facing side with the elements to be injected into the plasma. By hitting the thin film from behind, the laser beam generates and heats a plasma which expands, blows off and partially melts and vaporizes the coating ${ }^{35}$. This so-called "glass side" ablation mechanism depends on the laser energy density, the thickness and the optical properties of the coating as well as the thermodynamic properties of the coating material. In contrast to the direct "film side" ablation, the ablation from the glass side has the advantage of lower required laser pulse energies for an entire removal of the coating ${ }^{29,30}$ and leads to a higher particle yield ${ }^{24}$. The ablated particle beam consists of atoms, clusters, macro-particles, ions and electrons, strongly depending on the laser energy density and the coating thickness. Since charged particles cannot reach the plasma due to deflection by strong magnetic fields in fusion devices, only atoms as well as neutral clusters and macroparticles will reach the plasma. Inside the plasma, the neutral particles become ionized and emit radiation that can be detected by X-ray, XUV or visible spectrometers and cameras ${ }^{36}$. These measurements allow, together with simulations, to determine transport properties, such as impurity transport time, diffusion coefficient or convection velocity.

The paper firstly describes the experimental setup of the LBO system at W7-X, consisting of the laser, the optical components, the observation elements as well as the glass target system. In the second part, first experimental results are discussed including the evolution of the emission of several $\mathrm{Fe}$ ionization stages after an Fe injection. Finally, a short summary is given. 


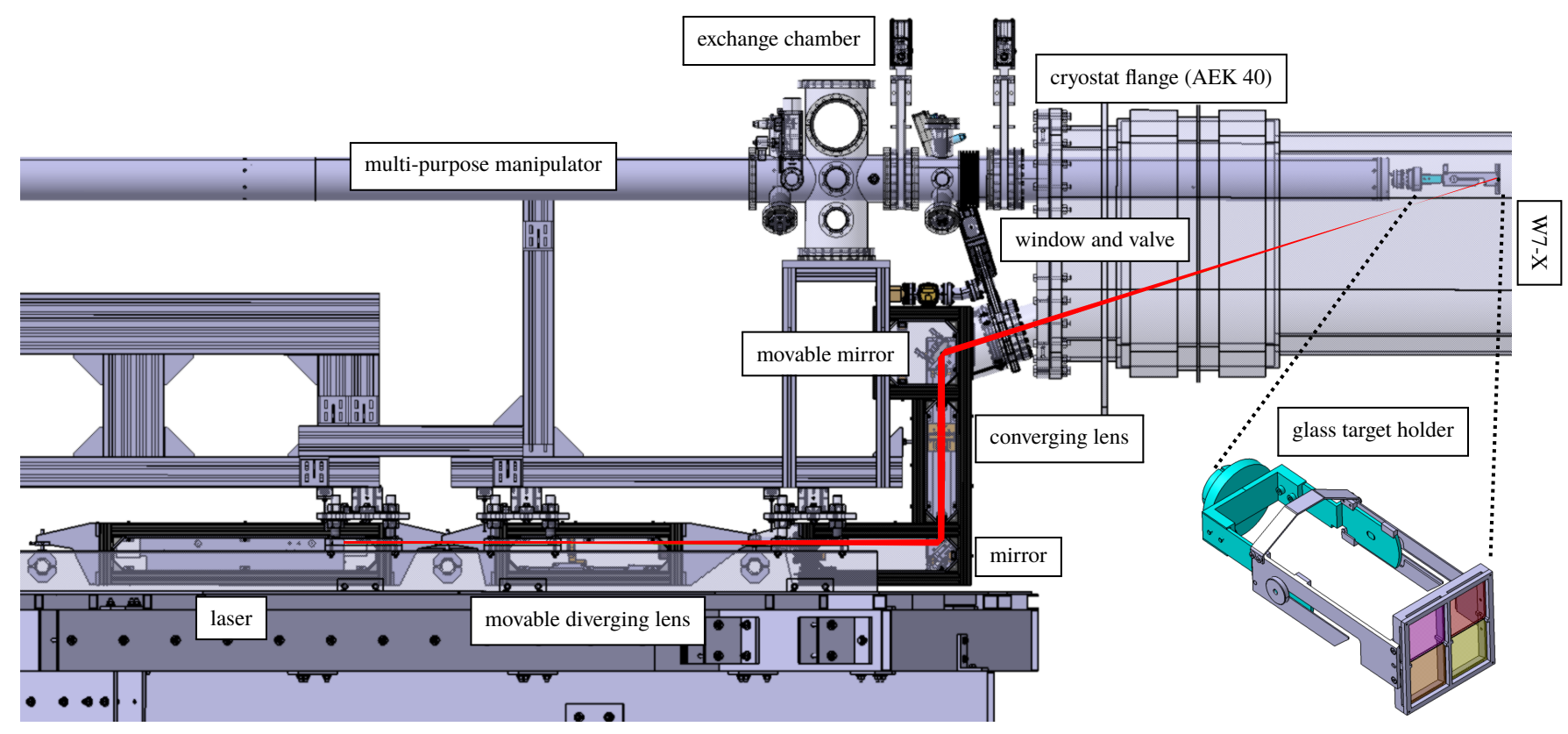

Figure 1. Schematic side view of the experimental setup with the result of the ray tracing calculation (red beam). The laser blow-off system is installed below the multi-purpose manipulator which is used for the positioning of the glass target holder inside the torus. The glass target holder, enlarged in the lower right corner, mounts 4 different glass targets at the same time.

\section{EXPERIMENTAL SETUP}

The LBO system of W7-X consists of a laser, an optical system and a glass target holder which can be mounted on the multi-purpose manipulator ${ }^{37}$ situated at a toroidal angle of $200.7^{\circ}$ on a cryostat flange (AEK 40), see figure 1. A Qswitched Nd:YAG laser $(1064 \mathrm{~nm})$ is used for the thin film ablation and can be operated in single pulse mode or in a burst mode (up to $20 \mathrm{~Hz}$ ) and has a pulse energy of $1 \mathrm{~J}$ within $6 \mathrm{~ns}$. Furthermore, a co-linear, continuous laser in the visible wavelength range $(650 \mathrm{~nm}, 5 \mathrm{~mW})$ indicates the location of the infrared beam.

The optical system is designed for both laser wavelengths while the transmissivity and reflectivity of the lenses and mirrors are optimized for the wavelength of $1064 \mathrm{~nm}$. The laser beam path from the laser output to the vacuum window is encapsulated for safety reasons which prevents stray radiation from escaping into the torus hall. The main purpose of the optical system is to guide the laser beam into the vacuum vessel and to adjust the spot diameter and the position on the glass target between two laser pulses. This allows to vary the ablated amount of material, to perform several pulses per plasma program and even to aim on different glass plates with different coatings. The design of the optical system is based on ray-tracing calculations, shown in figure 1 , that consider the refractive index, as well as the shape of lenses and assumes geometrical optics. A diverging negative meniscus lens ( $-500 \mathrm{~mm}$ focal length, broadband anti-reflection coating, $\mathrm{N}$ BK7) is installed on a remotely controllable linear stage just behind the laser beam exit. The lens widens up the laser beam and reduces the laser energy density on all optical components such that the laser damage threshold is not reached along the entire optical path. Furthermore, the remote movement of this lens enables the variation of the spot diameter on the glass target from 0.1 to $4 \mathrm{~mm}$. After the beam redirection with a mirror (broadband dielectric high-reflection coating), a fixed converging lens (1000 $\mathrm{mm}$ focal length, broadband anti-reflection coating, N-BK7) focuses the laser beam before the glass target, see figure 1 . Hence, the laser energy density behind the glass targets is low. This prevents damages on the torus wall when the laser beam passes through an ablated spot. After the converging lens, the laser beam is redirected with a steerable mirror (broadband dielectric high-reflection coating) and passes through a vacuum window into the torus on the glass targets. The remote steering of this mirror by means of piezo motors is fast enough to ensure a spot movement on the glass target of $5 \mathrm{~mm}$ within $50 \mathrm{~ms}$. Hence, the movement is fast enough that each laser pulse fired with a repetition rate of $20 \mathrm{~Hz}$ hits a non-ablated area on the coated glass. The piezo motors are able to work under the influence of the magnetic field which is estimated to about $100 \mathrm{mT}$ at the position of the motors ${ }^{38}$. In practice, the pre-calculated ray-tracing and the real optical path together with the estimated and measured spot diameter on the glass target are in very good agreement.

The glass target holder mounts 4 quartz glass plates (Corning $^{\circledR}$ HPFS $^{\circledR} 7980-5 f, 45 \times 45 \times 2 \mathrm{~mm}^{3}$ ), see figure 2 which were coated in vacuum devices ${ }^{39}$ with a thickness of 2 to $5 \mu \mathrm{m}$ using different techniques ${ }^{40}$, namely magnetron sputtering $(\mathrm{C}, \mathrm{Fe}, \mathrm{Mo}, \mathrm{W})$, thermal evaporation $(\mathrm{Al})$ and electron beam evaporation (Ti). The glass target holder can be inserted in the exchange chamber of the multi-purpose manipulator. Before dedicated LBO experiments, the manipulator head is positioned in the equatorial plane $645 \mathrm{~mm}$ away from the last closed flux surface (assuming the standard reference magnetic 


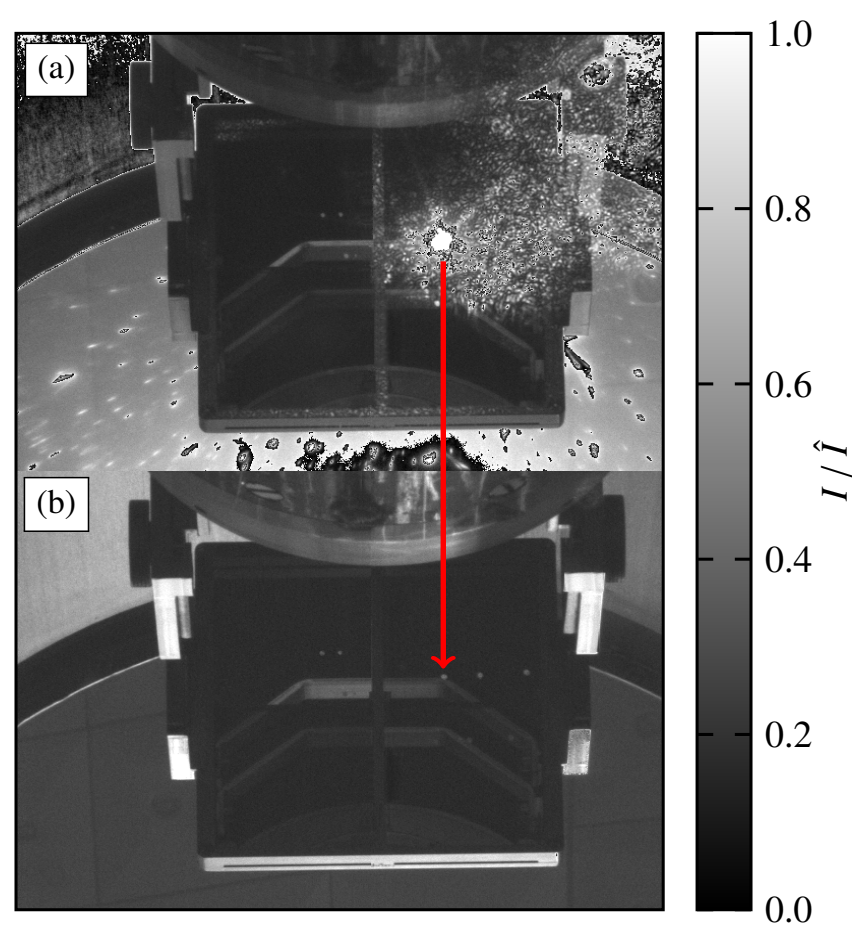

Figure 2. Snapshot of the mounted glass target holder which is inserted in the torus during (a) and after (b) the laser ablation. The visible emission of the plasma shines through the ablated spots.

field configuration, EJM $)^{41}$.

The whole laser blow-off system is remotely controllable. The timing of the laser pulses and steering operations are triggered by the W7-X MDSplus system. The amount of laser pulses and their timing are set in advance and automatically executed during the plasma pulse. The success of the whole ablation process was in-situ visually confirmed and recorded with a monochromatic charge-coupled device (CCD) camera, see figure 2. Furthermore, the camera was used to aim the laser spot on the desired location on the glass targets. A lightemitting diode (LED) supports the aiming by illuminating the glass targets. Both, the camera and the LED, are installed behind the movable mirror.

\section{RESULTS AND DISCUSSION}

Several experiments with more than 300 impurity injections in total were performed during the 2017 campaign of W7-X to verify the design goals of the new laser blow-off system and to perform first impurity transport studies. Different tracer materials $(\mathrm{Al}, \mathrm{C}, \mathrm{Fe}, \mathrm{Mo}, \mathrm{Si}, \mathrm{Ti}, \mathrm{W})$ were injected into helium and hydrogen plasmas heated by electron cyclotron resonance heating $(\mathrm{ECRH})$ in the range of $1-5 \mathrm{MW}$ and an electron density of $0.5-8 \times 10^{19} \mathrm{~m}^{-3}$ (helium) and $1-2.5 \times 10^{19} \mathrm{~m}^{-3}$ (hydrogen). However, the main injected tracer material was Fe because the available spectrometers are sensitive to the specific lines of different Fe ionization states, leading to a high overall diagnostic coverage. Furthermore, Fe

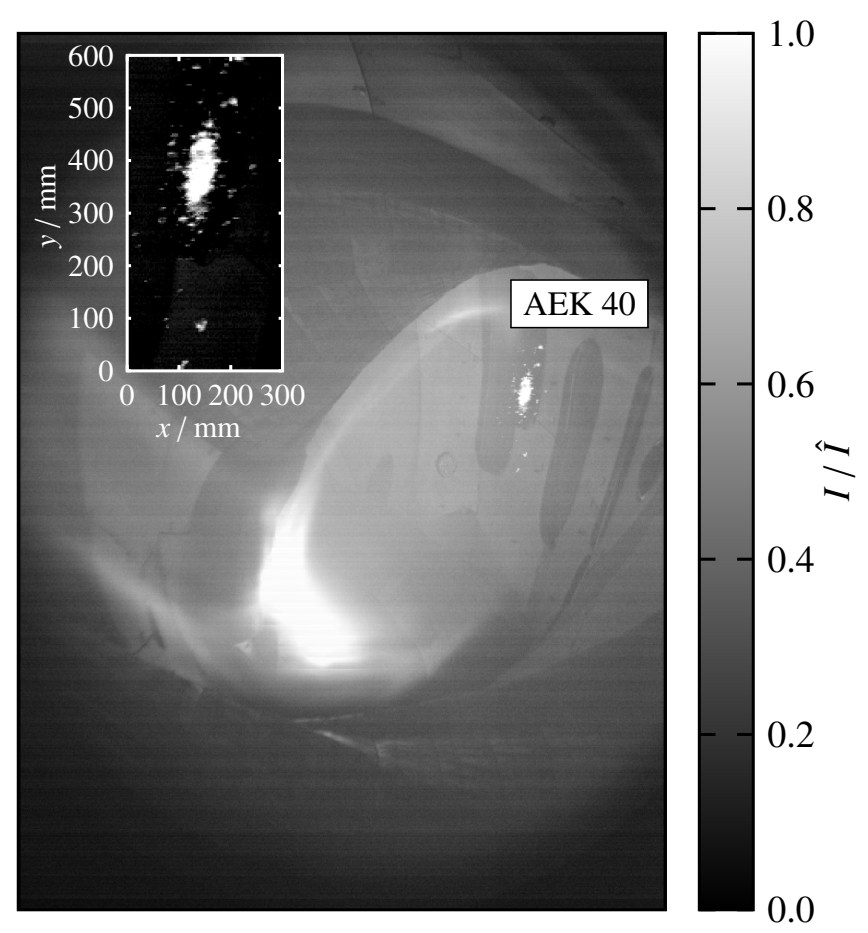

Figure 3. Cross section picture taken by means of the video diagnostics (visible spectral range) inside the torus during the LBO injection $(\mathrm{Fe})$ and a zoom of the emission pattern with spatial information for the experimental program 20171109.018.

as a mid- $Z$ material is suitable for the observed core electron temperatures between 1 and $5 \mathrm{keV}$. These experiments were conducted in different magnetic field reference configurations, namely the standard (EJM), high-mirror (KJM) and high-iota (FTM) configuration ${ }^{41}$. The injected particles and their influence on a manifold parameter set have been observed by a set of comprehensive diagnostics. In particular, the video diagnostics system ${ }^{42}$ was used to monitor the LBO injection. The bright pattern in the upper right corner of figure 3, presents the emission of lower ionization stages of injected particles in the visible spectral range. From these images, the size distribution of the injected amount of material can roughly be estimated. The large coherent pattern can be assigned to the well localized deposition of atoms, whereas the smaller spots in the vicinity present the clusters and macro-particles which are more broadly distributed.

Although the emission from the LBO injection is well observable, the global plasma parameters remain essentially constant and no strong perturbation by the impurity injections were observed. Figure 4 shows exemplarily the temporal evolution of the ECRH power (a), the plasma energy measured by the diamagnetic loop (b) and the emission of different ionization stages in the UV and X-ray spectral range for five massive LBO Fe injections (c). The plasma energy, which was analyzed similarly to the previous campaign ${ }^{43}$, decreases by only about 5\% after the injection. The emission intensity in the UV and X-ray spectral range shortly increases considerably after the LBO injection and is much larger than the emission by in- 

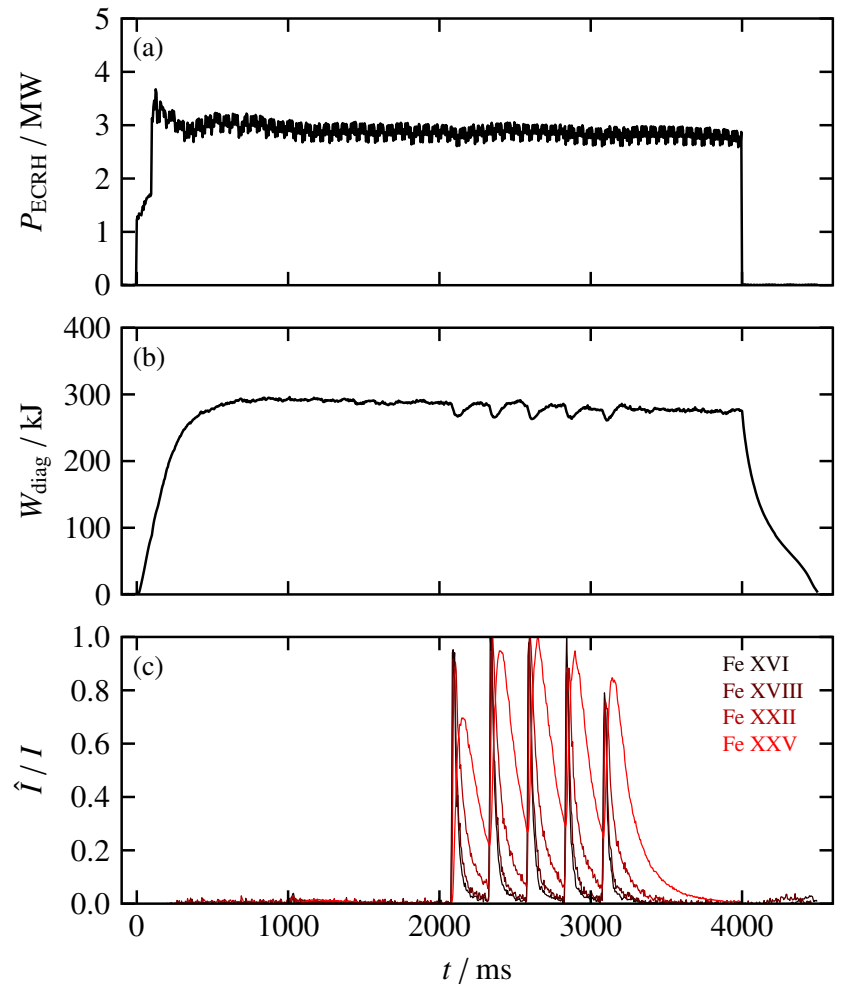

Figure 4. Time traces of electron cyclotron resonance heating power (a), plasma energy (b) and normalized intensities (c) for the ionization stages Fe XVI $(36.1 \mathrm{~nm})$, Fe XVIII $(9.4 \mathrm{~nm})$, Fe XXII $(11.7 \mathrm{~nm})$ and Fe XXV $(0.19 \mathrm{~nm})$, respectively, for the experimental program 20171123.036 .

trinsic impurities and the noise level. For this example, $5 \mu \mathrm{m}$ thick Fe layers were ablated with a spot diameter of $4 \mathrm{~mm}$ leading to an estimated amount of injected particles of about $1 \times 10^{18}$ even though just a fraction of these ablated particles reach the plasma. This example of massive repetitive injections demonstrates that an injection of tracer ions with the new laser blow-off system only has a weak effect on global plasma parameters. However, clear signals were measured that allow further analysis, e.g., the determination of impurity transport times.

Figure 5 illustrates a zoom of the intensity of one LBO injection. The emission of the lower ionization stages (Fe XVI, Fe XVIII and Fe XXII) is measured by means of a high-efficiency extreme ultraviolet overview spectrometer $(\mathrm{HEXOS})^{44}$ whereas a high resolution X-ray imaging spectrometer (HR-XIS) ${ }^{45,46}$ measures the emission for the heliumlike Fe XXV. After an impurity injection and assuming no other impurity sources or disturbing terms like MHD instabilities, the radial profile of the impurity density $n(r, t)$ exponentially decays with

$$
n(r, t)=\sum_{k} A_{k} n_{k}(r) \exp \left(-\frac{t}{\tau_{k}}\right),
$$

where $A_{k}, n_{k}, r, t$ and $\tau_{k}$ are constant factors, the profile of the eigenfunctions, the radius, the time and the eigenvalues

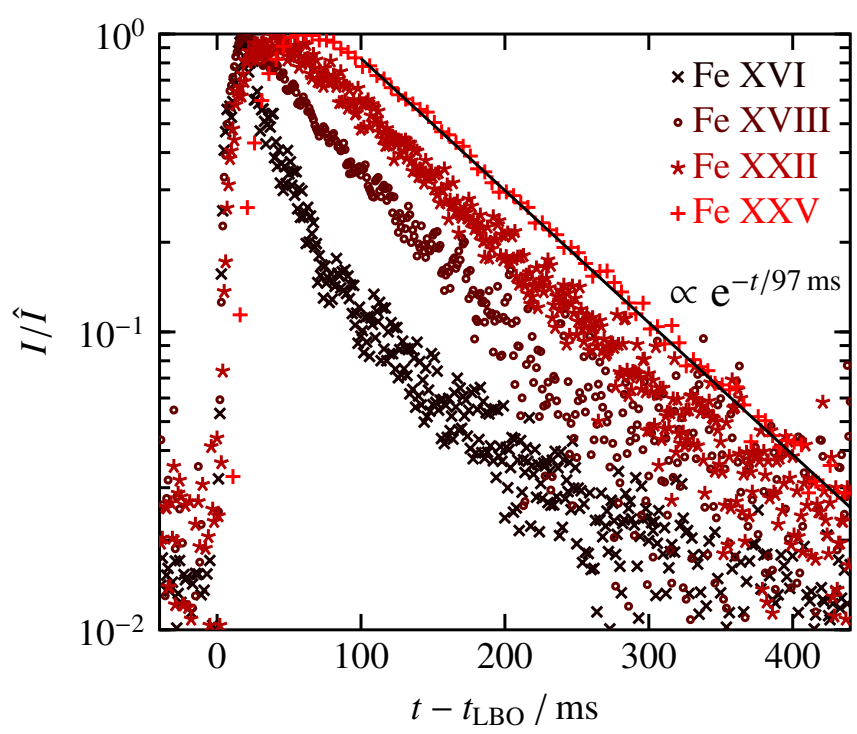

Figure 5. Normalized temporally resolved intensities for the ionization stages Fe XVI $(36.1 \mathrm{~nm}, \times)$, Fe XVIII $(9.4 \mathrm{~nm}, \circ)$, Fe XXII $(11.7 \mathrm{~nm}, \star)$ and Fe XXV (+), respectively, after a LBO Fe injection and the exponential fit for the decaying part of the Fe XXV intensity with a time constant of $97 \mathrm{~ms}$ which is an estimation for the impurity transport time for the experimental program 20171109.018.

of the radial transport equation whereas the slowest eigenvalue $(k=0)$ is here defined as the impurity transport time, respectively $^{47}$. Equation 1 is a general and complete solution for the radial transport of impurities concerning diffusion and convection which is assumed to be the same for all ionized states. The lower ionization stages peak earlier compared to higher ionization stages because the injected Fe particles are ionized stepwise and transported from the plasma edge to the plasma core. In the plasma core, the higher temperatures yield higher ionization levels which show a slower temporal behavior. The decay phase of the spectral line intensities of lower ionization stages can be divided into an initial phase of fast decay (within the first $100 \mathrm{~ms}$ after the peak) and a slow decay phase in the time range starting at $100 \mathrm{~ms}$ after the injection. The fast decay phase is the result of ionization of impurity atoms into higher stages and is different for the various lowest ionization stages. The slow decay phase is similar for all ionization stages since it is connected to transport quantities rather then atomic processes and can, thus, be attributed to the impurity transport time $\tau_{0}$.

Thus, after $\tau_{0}$ becomes the dominant timescale, the evaluation of the decaying part of the helium-like or hydrogen-like Fe spectral line intensity yield information about the confinement property of the impurity species. For the here considered example we find $\tau_{0}=97 \pm 2 \mathrm{~ms}$. Compared to that, the energy confinement time $\tau_{\mathrm{e}}$, calculated from the ratio of the plasma energy and the heating power, amounts to about $\tau_{\mathrm{e}}=124 \mathrm{~ms}$ and decreases immediately after the LBO injection to about $\tau_{\mathrm{e}}=118 \mathrm{~ms}$. This reduction of about $3 \%$ is caused by the decreasing plasma energy following the impurity injection. Other impurity transport studies show similar 
results regarding the transport time which is in the same order of magnitude ${ }^{48}$.

To account for the impurity transport time in the order of $100 \mathrm{~ms}$, the time difference between two laser pulses was generally set to about $500 \mathrm{~ms}$ in the case of multiple impurity injections. Hence, the measured temporal evolution of the emission line intensities from excited tracer ions can be analyzed separately without overlapping each other. Nevertheless, a repetition rate of $20 \mathrm{~Hz} \mathrm{LBO}$ injection was also tested to demonstrate the capabilities of the new laser blow-off system. In that experimental program, $\mathrm{W}$ was massively injected into the plasma which ultimately led to a radiative collapse and terminated the plasma.

\section{SUMMARY}

In summary, the LBO system was successfully installed at the Wendelstein 7-X stellarator and operated in the second operation phase (OP1.2a) in 2017. The system reached the design goals, worked routinely and conducted more than 300 injection of 7 different materials. This way, non-intrinsic and non-recycling tracer ions are injected into the plasma in a controlled manner for transport studies. The amount of injected impurities can be controlled by remotely changing the spot diameter and the spot position on the glass target between two laser shots. During first LBO experiments in a target plasma with low densities and $5 \mathrm{MW}$ of ECRH heating power, the impurity transport time was estimated to be about $100 \mathrm{~ms}$ whereas the energy confinement time was approximately $120 \mathrm{~ms}$ for a typical experiment program. The plasma energy was maximum decreased by $5 \%$, thereby confirming that the LBO impurity injection did not significantly perturb the plasma while providing sufficient impurities for the detailed measurement by X-ray to XUV diagnostics. The new laser blow-off system enables impurity injection for impurity transport studies providing experimental data for transport analysis codes.

\section{ACKNOWLEDGMENTS}

The authors thank Klaus Höchel and Raphael Rataj for technical support during the assembly phase.

This work has been carried out within the framework of the EUROfusion Consortium and has received funding from the Euratom research and training programme 2014-2018 under grant agreement No 633053. The views and opinions expressed herein do not necessarily reflect those of the European Commission.

\section{REFERENCES}

${ }^{1}$ H. Maassberg, C. D. Beidler, and E. E. Simmet, Plasma Physics and Controlled Fusion 41, 1135 (1999).

${ }^{2}$ P. Helander, S. L. Newton, A. Mollén, and H. M. Smith, Phys. Rev. Lett. 118, 155002 (2017).
${ }^{3}$ H. Nozato, S. Morita, M. Goto, A. Ejiri, and Y. Takase, Review of Scientific Instruments 74, 2032 (2003).

${ }^{4}$ S. Morita, M. Goto, S. Muto, H. Y. Zhou, C. F. Dong, and LHD Experiment Group, Fusion Science and Technology 58, 91 (2010).

${ }^{5}$ D. Hildebrandt, J. S. Bakos, G. Petravich, H. Badalec, and K. Jakubka, Review of Scientific Instruments 60, 547 (1989).

${ }^{6}$ E. S. Marmar, J. L. Cecchi, and S. A. Cohen, Review of Scientific Instruments 46, 1149 (1975).

${ }^{7}$ S. A. Cohen, J. L. Cecchi, and E. S. Marmar, Phys. Rev. Lett. 35, 1507 (1975).

${ }^{8}$ R. Isler, Nuclear Fusion 24, 1599 (1984).

${ }^{9}$ D. Pasini, M. Mattioli, A. Edwards, R. Giannella, R. Gill, N. Hawkes, G. Magyar, B. Saoutic, Z. Wang, and D. Zasche, Nuclear Fusion 30, 2049 (1990).

${ }^{10}$ R. Burhenn, A. Weller, W7-AS Team, NI Group, and The ECRH Group, Review of Scientific Instruments 70, 603 (1999).

${ }^{11}$ E. Scavino, J. S. Bakos, R. Dux, H. Weisen, and TCV Team, Plasma Physics and Controlled Fusion 45, 1961 (2003).

${ }^{12}$ R. Burhenn, Y. Feng, K. Ida, H. Maassberg, K. McCarthy, D. Kalinina, M. Kobayashi, S. Morita, Y. Nakamura, H. Nozato, S. Okamura, S. Sudo, C. Suzuki, N. Tamura, A. Weller, M. Yoshinuma, and B. Zurro, Nuclear Fusion 49, 065005 (2009).

${ }^{13}$ N. T. Howard, M. Greenwald, and J. E. Rice, Review of Scientific Instruments 82, 033512 (2011).

${ }^{14}$ B. Zurro, E. Hollmann, A. Baciero, M. Ochando, F. Medina, K. McCarthy, E. Blanco, E. de la Cal, D. Carralero, M. Pedrosa, and TJ-II Team, Nuclear Fusion 51, 063015 (2011).

${ }^{15}$ M. Kissick, E. Fredrickson, J. Callen, C. Bush, Z. Chang, P. Efthimion, R. Hulse, D. Mansfield, H. Park, J. Schivell, S. Scott, E. Synakowski, G. Taylor, and M. Zarnstorff, Nuclear Fusion 34, 349 (1994).

${ }^{16}$ K. W. Gentle, R. V. Bravenec, G. Cima, H. Gasquet, G. A. Hallock, P. E. Phillips, D. W. Ross, W. L. Rowan, A. J. Wootton, T. P. Crowley, J. Heard, A. Ouroua, P. M. Schoch, and C. Watts, Physics of Plasmas 2, 2292 (1995).

${ }^{17}$ F. Ryter, R. Neu, R. Dux, H.-U. Fahrbach, F. Leuterer, G. Pereverzev, J. Schweinzer, J. Stober, W. Suttrop, ASDEX Upgrade Team, F. D. Luca, A. Jacchia, and J. Kinsey, Nuclear Fusion 40, 1917 (2000).

${ }^{18}$ K. McCormick, Review of Scientific Instruments 56, 1063 (1985).

${ }^{19}$ A. Pospieszczyk and G. G. Ross, Review of Scientific Instruments 59, 1491 (1988).

${ }^{20}$ J. S. Bakos, G. Burger, I. B. Földes, P. E. Giese, P. N. Ignácz, G. Petravich, J. Szigeti, and S. Zoletnik, Plasma Physics and Controlled Fusion 31, 693 (1989).

${ }^{21}$ D. Michaud, G. G. Ross, E. Haddad, H. H. Mai, A. Pospieszczyk, and J. P. St-Germain, Review of Scientific Instruments 63, 5698 (1992).

${ }^{22}$ S. Sasaki, S. Takamura, Y. Uesugi, Y. Ohkouchi, and K. Kadota, Review of Scientific Instruments 64, 2277 (1993).

${ }^{23}$ M. Bruchhausen, R. Burhenn, M. Endler, G. Kocsis, A. Pospieszczyk, S. Zoletnik, and W7-AS Team, Plasma Physics and Controlled Fusion 46, 489 (2004).

${ }^{24}$ J. F. Friichtenicht, Review of Scientific Instruments 45, 51 (1974).

${ }^{25}$ N. G. Utterback, S. P. Tang, and J. F. Friichtenicht, The Physics of Fluids 19, 900 (1976).

${ }^{26}$ D. Manos, D. Ruzic, R. Moore, and S. Cohen, Journal of Vacuum Science and Technology 20, 1230 (1982).

${ }^{27}$ S. Mattoo, L. Wirtz, A. Pospieszczyk, and B. Schweer, Nuclear Instruments and Methods in Physics Research Section B: Beam Interactions with Materials and Atoms 124, 579 (1997).

${ }^{28}$ A. B. Bullock, P. R. Bolton, and F. J. Mayer, Journal of Applied Physics 82, 1828 (1997)

${ }^{29}$ D. Bartl, A. Michalowski, M. Hafner, A. Letsch, S. Nolte, and A. Tünnermann, Applied Physics A 110, 227 (2013).

${ }^{30}$ G. Heise, M. Englmaier, C. Hellwig, T. Kuznicki, S. Sarrach, and H. P. Huber, Applied Physics A 102, 173 (2011).

${ }^{31}$ M. Domke, L. Nobile, S. Rapp, S. Eiselen, J. Sotrop, H. P. Huber, and M. Schmidt, Physics Procedia 56, 1007 (2014)

${ }^{32}$ J. Bohandy, B. F. Kim, and F. J. Adrian, Journal of Applied Physics 60, 1538 (1986).

${ }^{33}$ F. H. Karg, Solar Energy Materials and Solar Cells 66, 645 (2001).

${ }^{34}$ S. Zoppel, H. Huber, and G. Reider, Applied Physics A 89, 161 (2007). 
${ }^{35}$ S. Amoruso, R. Bruzzese, N. Spinelli, and R. Velotta, Journal of Physics B: Atomic, Molecular and Optical Physics 32, R131 (1999).

${ }^{36} \mathrm{H}$. Thomsen, A. Langenberg, D. Zhang, G. Bertschinger, C. Biedermann, W. Biel, R. Burhenn, B. Buttenschön, K. Grosser, R. König, M. Kubkowska, O. Marchuk, N. Pablant, L. Ryc, T. Pedersen, and W7X Team, Journal of Instrumentation 10, P10015 (2015).

${ }^{37}$ D. Nicolai, V. Borsuk, P. Drews, O. Grulke, K. Hollfeld, T. Krings, Y. Liang, C. Linsmeier, O. Neubauer, G. Satheeswaran, B. Schweer, and G. Offermanns, Fusion Engineering and Design 123, 960 (2017).

${ }^{38}$ T. Andreeva and E. Harmeyer, "Das magnetische Streufeld in der Maschinenhalle von Wendelstein 7-X," (IPP 13/2) (Garching: Max-Planck-Institut für Plasmaphysik, 2003).

${ }^{39}$ S. Peglow, M.-M. Pohl, A. Kruth, and V. Brüser, The Journal of Physical Chemistry C 119, 563 (2015).

${ }^{40}$ J. Harhausen, R. P. Brinkmann, R. Foest, M. Hannemann, A. Ohl, and B. Schröder, Plasma Sources Science and Technology 21, 035012 (2012).

${ }^{41}$ T. Andreeva, "Vacuum Magnetic Configurations of Wendelstein 7-X," (IPP III/270) (Garching: Max-Planck-Institut für Plasmaphysik, 2002).

${ }^{42}$ G. Kocsis, T. Baross, C. Biedermann, G. Bodnár, G. Cseh, T. Ilkei, R. König, M. Otte, T. Szabolics, T. Szepesi, and S. Zoletnik, Fusion Engineering and Design 96-97, 808 (2015).
${ }^{43}$ K. Rahbarnia, H. Thomsen, U. Neuner, J. Schilling, J. Geiger, G. Fuchert, T. Andreeva, M. Endler, D. Hathiramani, T. Bluhm, M. Zilker, B. B. Carvalho, A. Werner, and W7-X Team, "Diamagnetic energy measurement during the first operational phase at the Wendelstein 7-X stellarator," (2018), accepted in Nuclear Fusion.

${ }^{44}$ W. Biel, G. Bertschinger, R. Burhenn, R. König, and E. Jourdain, Review of Scientific Instruments 75, 3268 (2004)

${ }^{45} \mathrm{G}$. Bertschinger, W. Biel, H. Jaegers, and O. Marchuk, Review of Scientific Instruments 75, 3727 (2004).

${ }^{46}$ A. Langenberg, J. Svensson, H. Thomsen, O. Marchuk, N. A. Pablant, R. Burhenn, and R. C. Wolf, Fusion Science and Technology 69, 560 (2016).

${ }^{47}$ F. H. Seguin, R. Petrasso, and E. S. Marmar, Phys. Rev. Lett. 51, 455 (1983).

${ }^{48}$ A. Langenberg, N. Pablant, Th. Wegner, P. Traverso, O. Marchuk, T. Bräuer, G. Fuchert, S. Bozhenkov, E. Pasch, B. Geiger, A. Pavone, J. Svensson, J. Alonso, R. Burhenn, R. Wolf, and the W7-X Team, "Prospects of X-ray Imaging Spectrometers for Impurity Transport: Recent Results from the Stellarator Wendelstein 7-X," (2018), accepted in Review of Scientific Instruments. 